\title{
Worksite Wellness Programs on the USA-Mexico Border
}

\author{
Sharon E. Thompson \\ The University of Texas at El Paso
}

\begin{abstract}
Worksite wellness programs (WWPs) have the potential to reduce absenteeism, increase productivity, and improve employee attitudes and job performance with distinct economic benefits. The purpose of this study was to collect data about WWPs in the manufacturing industry in El Paso, Texas and formulate recommendations to increase the use of WWPs to protect, promote and improve the health of this workforce. RESULTS: The results of this study indicate a lack of worksite wellness programs in small to medium-sized manufacturers. Worksite size was a strong indicator of the number of worksite wellness program activities that were offered. Large worksites were more likely to offer more activities than small or medium sized worksites. This difference is perhaps due to a greater availability of resources (such as money, facilities, staff, etc.). Administrators perception of worksite wellness programs is of particular interest. The main benefit reported by respondents with existing WWPs was improved health and decreased health problems (83\%) and decreased health care costs (70\%). Conversely, the main barrier perceived by administrators at sites lacking WWPs was that implementation was too costly (38\%). Cost may be perceived as the major barrier because companies without wellness programs may only consider the start-up costs. Information on the long-term economic benefits of WWPs could alter this perception. Future efforts to initiate worksite wellness programs must be tailored to meet the needs of small and medium sized companies in this border community.
\end{abstract}

(c) 2003 Californian Journal of Health Promotion. All rights reserved.

Keywords: worksite wellness, border, health promotion, Texas

\section{Background}

Currently, employers pay approximately onethird of the national health care bill (Reardon, 1998, Ahrens, 1995). Preventive measures are being employed by businesses in an effort to contain the escalating costs of employee illness. In the United States, an estimated 110 million persons are employed, and spend approximately one-third of their time at the job site (Reardon, 1998; Stokols, Pelletier \& Fielding, 1995). Because eighty-five percent of the adult population of the US is employed, the worksite is an ideal setting to implement a health promotion program. Worksite wellness programs have the potential to improve the health of the employee and their dependents. The most common health promotion activities include: smoking cessation, stress management, health risk assessment, cardiovascular risk reduction, weight control, and exercise and fitness (Kizer, Pelletier \& Fielding, 1995).
Numerous studies have concluded that properly implemented WWPs can improve employee health outcomes and have economic benefits (Wellness Councils of America, 1994, Stead, 1994). Worksite wellness programs have the potential to reduce absenteeism, increase productivity, and improve employee attitudes and job performance (Conners, 1992; Fowler \& Risner, 1994; Mason, 1992). Twenty-four peerreviewed studies published from 1980 to 1991 concluded that positive health outcomes result from the implementation of worksite wellness programs (Kizer, 1995). An additional twentyfour peer-reviewed studies published from 1991 to 1993 indicated positive health outcomes relative to smoking cessation, weight loss, and the reduction of coronary heart disease risk factors (Kizer, 1995).

The population of El Paso County is approximately 679,622 (US Census, 2002). The 
El Paso community reflects the health concerns of other Latino populations in the United States. "Activity limitation, infectious disease, chronic disease, and poor nutritional status” are health concerns of the Latino community (CarterPokras, 1994, p. 45). El Paso has been characterized as rapidly expanding, predominantly Hispanic, primarily young, and economically disadvantaged (Michie, 1992). The manufacturing industry employs 18.6 percent of the El Paso workforce, yet there is no information about the worksite wellness programs implemented to address the health concerns of this vulnerable population (City of El Paso, 1996).

\section{Methodology Population \\ Selection and Recruitment of Study}

In order to identify current worksite wellness programs in practice, determine the types of activities available, and identify perceived benefits or barriers to the implementation of worksite wellness programs in El Paso, Texas, a survey of the manufacturing industry of El Paso was conducted. The manufacturing industry was selected for the study because it employees $18.6 \%$ of the labor force of El Paso (City of El Paso, 1996). The target population was all manufacturers in El Paso county, registered with the El Paso Chamber of Commerce. The questionnaire with a letter inviting participation and assuring confidentiality was sent to the contact person of these 577 manufacturing companies. The instrument was approved by The Committee for the Protection of Human Subjects at The University of Texas Houston Health Science Center, A self-addressed stamped envelope was enclosed with the instrument in order to increase the response rate.

\section{Instrument Development}

The survey was developed after a review of the literature on worksite wellness programs. The first question of the survey requested information about the existence of a worksite wellness program and the size of the company. The questionnaire was then divided into two parts. Manufacturers without a WWP were asked to complete six questions covering the reasons the company did not have a WWP, if their company would be interested in implementing a WWP, the reasons for their interest, and what specific activities were of particular interest (Chenoweth, 1987). The second part of the survey requested manufacturers with an existing WWP complete a series of questions regarding the reasons for implementation, types of activities offered, and utilization of nonprofit services.

\section{Data Analysis}

Data from completed questionnaires were entered into an SPSS database. Manufacturers were divided into small, medium, and large companies based on the number of employees. Small companies were defined as manufacturers employing less than 50 persons. Companies with 50 to 299 employees were defined as medium sized companies. Large companies were defined as those with over 300 employees. An analysis of variance (ANOVA) was performed to determine if a significant difference existed between the size of the worksite and the number of programs offered. The alpha level set for this ANOVA was $\leq 0.05$.

\section{Results}

Sixty-four manufacturers responding to the survey with a response rate of $11.8 \%$. In the thirty worksites with an active program, a total of 390 activities were reported as being offered. Worksite wellness program activities were categorized as healthy lifestyle education, screenings, monitoring and follow-up, safety promotion and accident prevention, and employee assistance programs (Table 1). Under the category of healthy lifestyle education, $80 \%$ offered activities on lifting properly. Diabetes screening (63\%), immunization (e.g., tetanus booster), (53\%) and heart disease risk identification (50\%) were the most frequently offered screenings, monitoring, and follow-up activities. The vast majority of activities offered by companies fell into the safety promotion and accident prevention category. The majority of the companies offered emergency medical treatment and first aid (93\%), on-the-job safety instruction (86\%), and "right-to-know" education (identify potentially hazardous substances) (75\%). Of the employee assistance programs (EAPs), alcohol and other drug abuse 
counseling was offered by $73 \%$ of the companies. Less than half of these companies offered EAPs covering preparing for retirement, coping with depression, and domestic counseling.
The mean number of activities varied significantly with the size of the worksite $(\mathrm{F}=$ 7.97, $\mathrm{df}=2,27, \mathrm{p} \leq 0.0019)$. Large worksites offered significantly more activities than small or medium sized worksites. The mean number of activities for small worksites was 10 , medium 6 , and large 15 (Table 2).

Table 1

Type of Worksite Wellness Activity Respondents Offered

\begin{tabular}{|c|c|c|}
\hline Activity & $\begin{array}{c}\text { Number of } \\
\text { Companies Offering } \\
\text { Activities }(\mathrm{N}=30)\end{array}$ & $\begin{array}{c}\text { Percent of } \\
\text { Companies Offering } \\
\text { Activities (\%) }\end{array}$ \\
\hline \multicolumn{3}{|l|}{ Healthy Lifestyle Education } \\
\hline Exercise and physical fitness & 19 & 63 \\
\hline Lifting properly & 24 & 80 \\
\hline Nutrition & 15 & 50 \\
\hline Smoking cessation & 19 & 63 \\
\hline Stress management & 9 & 30 \\
\hline Weight control & 11 & 37 \\
\hline Drug awareness & 23 & 77 \\
\hline Other & 6 & 20 \\
\hline \multicolumn{3}{|l|}{ Screening, Monitoring, and Follow-Up } \\
\hline Cancer screening & 14 & 47 \\
\hline Diabetes screening & 19 & 63 \\
\hline Heart disease risk identification & 15 & 50 \\
\hline Hypertension screening & 11 & 37 \\
\hline Immunization & 16 & 53 \\
\hline Other & 6 & 20 \\
\hline \multicolumn{3}{|l|}{ Safety Promotion and Accident Prevention } \\
\hline Cardio pulmonary resuscitation (CPR) & 16 & 53 \\
\hline Choke-safety techniques & 17 & 57 \\
\hline Emergency medical treatment and first aid & 28 & 93 \\
\hline On-the-job safety instruction & 26 & 87 \\
\hline Right-to-know education & 23 & 77 \\
\hline Seat belt/shoulder strap usage & 7 & 23 \\
\hline Other & 5 & 17 \\
\hline \multicolumn{3}{|l|}{ Employee Assistance Program } \\
\hline Alcohol and other drug abuse counseling & 22 & 73 \\
\hline Coping with depression & 12 & 40 \\
\hline Domestic counseling & 12 & 40 \\
\hline Preparation for retirement & 13 & 43 \\
\hline Other & 2 & 7 \\
\hline
\end{tabular}

Note: These percentages do not sum to 100 as companies offered multiple activities. 
The thirty manufacturers with a worksite wellness program were asked to cite the reason or reasons for the implementation of their program (Table 3). The vast majority (83\%) of the respondents reported improving health as the reason for implementing a worksite wellness program. Well over half (70\%) of the companies cited both improving employee morale and decreasing health care costs as reasons.

Table 2

Mean Number of Activities Offered by Size of Worksite

\begin{tabular}{|l|c|c|}
\hline Size of Worksite & $\begin{array}{c}\text { Number of Worksites } \\
\text { (N=30) }\end{array}$ & $\begin{array}{c}\text { Mean Number of Activities } \\
\text { Offered }\end{array}$ \\
\hline Small & 6 & 10 \\
\hline Medium & 3 & 6 \\
\hline Large & 21 & 15 \\
\hline Total & 30 & 31 \\
\hline
\end{tabular}

Table 3

Reasons for Implementing a Worksite Wellness Program (WWP)

\begin{tabular}{|l|c|c|}
\hline \multicolumn{1}{|c|}{$\begin{array}{c}\text { Reason for Implementing a Worksite } \\
\text { Wellness Program (WWP) }\end{array}$} & $\begin{array}{c}\text { Number of Companies } \\
\text { (N=30) }\end{array}$ & $\begin{array}{c}\text { Percent of Companies } \\
\text { (\%) }\end{array}$ \\
\hline Improve health and decrease health problems & 25 & 83 \\
\hline Decrease health care costs & 21 & 70 \\
\hline Improve employee morale & 21 & 70 \\
\hline Improve productivity & 16 & 53 \\
\hline Response to employee interest or demand & 8 & 27 \\
\hline Desire to be part of an innovative trend & 7 & 10 \\
\hline Other & 3 & \\
\hline Total Responses & 101 & 23 \\
\hline
\end{tabular}

Note: Respondents were asked to "mark all that apply." These percentages do not sum to 100 as companies offered multiple reasons.

The thirty-four respondents without existing worksite wellness programs were asked to cite the reason or reasons why their worksite did not have a WWP (Table 4). The most common response, "Too costly," was cited by $38 \%$ of the respondents. Respondents also cited "employees do not show an interest" (35\%), other reasons (29\%), and "no need/employees healthy" (24\%). The "other" category was an open-ended question and allowed the respondent to express an individual response. Other reasons included: "too small an operation," "too many other issues to worry about," "uninformed," and "it is a useless proposition.” Four manufacturers (12\%) said they had not initiated WWP because they felt it would interfere with workday routine, would be too time consuming, they had high employee turnover, and it would be too difficult to implement. "No perceived benefits" was a reason reported to a much lesser extent (9\%). 
Table 4

Perceived Barriers to the Implementation of a Worksite Wellness Program

\begin{tabular}{|l|c|c|}
\hline \multicolumn{1}{|c|}{ Perceived Barriers } & Number of Companies (N=34) & Percent of Companies (\%) \\
\hline Too costly & 13 & 38 \\
\hline Employees do not show an interest & 12 & 35 \\
\hline Other & 10 & 29 \\
\hline No need/employees healthy & 8 & 24 \\
\hline $\begin{array}{l}\text { Interferes with workday routine/ } \\
\text { too time consuming }\end{array}$ & 4 & 12 \\
\hline High employee turnover & 4 & 12 \\
\hline Too difficult to implement & 4 & 12 \\
\hline No perceived benefits & 4 & 12 \\
\hline
\end{tabular}

Note: Respondents were asked to "mark all that apply.” These percentages do not sum to 100 as companies offered multiple reasons.

\section{Discussion}

Large worksite were much more likely to have WWPs. Worksite size also was a strong indicator of the number of worksite wellness program activities that were offered. Large worksites were more likely to offer more activities than small or medium sized worksites. This difference is perhaps due to a greater availability of resources (such as money, facilities, staff, etc.).

Employee's perception of worksite wellness programs is of particular interest. The main benefits reported by respondents with existing WWPs were improved health and decreased health problems (83\%) and decreased health care costs (70\%). Conversely, the main barriers perceived by the contact person at the worksite where there was no WWP, were that implementation was too costly (38\%). Cost may be perceived as the major barrier because companies without wellness programs may only consider the start-up costs. Understanding that, over the long term, WWPs might reduce costs, could alter this perception.

Of the thirty-four worksites without a WWP responding to the survey, only seven (21\%) were interested in starting a WWP. However, when asked in a follow-up question, "Which of the following activities would this company be interested in implementing?," 19 respondents (56\%) were interested in initiating one or more of the listed activities. Perhaps the idea of implementing a "worksite wellness program" is more daunting than merely initiating one or more activities.

Of the activities of interest, emergency medical treatment and first aid (75\%) and domestic counseling (70\%) ranked among the highest. The interest in emergency medical treatment and first aid may reflect an effort to treat occupational injuries and illnesses that have occurred in the past and continue to occur. Perhaps a properly implemented injury prevention program could limit the number and/or the extent of these occupational injuries. A finding that was not anticipated was the level of interest in domestic counseling. This may reflect a major concern involving finances and family problems that have traditionally not been included in many worksite wellness programs, but are highly pervasive problems in today's society.

The major limitation of this study is the lack of representation of worksites in the manufacturing industry of El Paso as evidence by the response rate of 11.8 percent. The low response rate in this study may indicate a lack of interest on the part of members of worksites in El Paso to participate in research involving wellness programs. However, the data collected provide a broad and interesting profile of the type of worksite wellness programs available in the manufacturing industry of El Paso, which 
accounts for approximately 19\% of the El Paso labor force.

\section{Conclusions and Recommendations}

The majority of decisions regarding worksite wellness programs tend to be made by top administrators and persons in personnel/human resources/benefits departments. In order to expand the implementation of WWPs in the El Paso region, efforts must be directed at reaching top administrative personnel with the pertinent information. This information should include the data on improved health outcomes of participants in WWPs. The results of the study indicate that cost is the major barrier to the implementation of WWPs. Particular emphasis should be placed on the economic benefits of WWPs.

The information disseminated to top-level administrators could also be improved by the development of more rigorous evaluation approaches to assess economic benefits and health outcomes of worksite wellness programs. Information on the anticipated cost-effectiveness of worksite wellness programs is necessary in order for administrators to make sound decisions. A wider array of measurement strategies is advised. Past evaluations have been very specific and measured particular health outcomes. Broader evaluations may include measurements in general health outcomes as a result of participation in WWPs.

The results of this study indicate a lack of worksite wellness programs in small to mediumsized manufacturers. Future efforts by organizations dedicated to improving health at the worksite need to be tailored to meet the needs of small and medium sized companies. Smaller companies generally offer fewer worksite wellness program activities. This is generally attributed to the lack of staff and financial resources (Stokols et al., 1995). It has been projected that these barriers will decrease with managed care legislation mandating worksite injury and illness prevention programs (Stokols et al., 1995).

There are many organizations dedicated to the promotion of health-related activities at the worksite. Small and medium sized companies in El Paso who perceive cost as the major barrier to the implementation of a WWP should utilize the services and resources provided by these organizations. For instance, The National Association of Governor's Councils on Physical Fitness and Sports is dedicated to the improvement of individuals health at the worksite (Prata, 1993). This organization offers creative worksite wellness program initiatives and developed a National Employee Health and Fitness Day.

In addition, the worksites of El Paso should utilize the services provided by the not-for-profit and voluntary agencies in the region. Not only does the local chapter of the American Cancer Society offer comprehensive cancer prevention and educational programs, but there is a program specifically designed to encourage and assist worksites in the development and implementation of their own WWP. These services are offered to worksites of any size and are free of charge or for a nominal fee. The El Paso Diabetes Association offers free educational programs to worksites and blood glucose screenings for a nominal fee. The local chapters of the American Cancer Society, American Heart Association, and American Lung Association offer similar preventive and educational services to worksites. Administrators interested in the improvement of employee health and the reduction of health related expenses can easily and affordably develop a worksite wellness program by utilizing services readily available in the El Paso community.

Worksite wellness programs must be designed to attract those employees with the greatest health concerns (Glasgow, McCaul \& Fisher, 1993). It is also necessary to recognize the differences of demographic and behavioral risk factors in order to increase participation among employees with high-risk health behaviors. These health initiatives must address the needs of El Paso's ethnically diverse population. Strategies must be incorporated to recruit this under-served group through the development of culturally and sensitive worksite wellness programs (Sorensen, Glasgow \& Corbett, 1990). 


\title{
References
}

Ahrens, R. (1995). The healthy workplace. 17(11), 77-86.

Carter-Pokras, O. (1994). Health profile. In C. W. Molina \& M. Aguirre-Molina (Eds.), Latino health in US: A growing challenge (pp. 45-79). Washington, DC: American Public Health Association.

Chenoweth, D. (1987). Planning health promotion at the worksite. New Jersey: Benchmark Press.

City of El Paso. (1996). City of El Paso economic development: Employment growth. Retrieved November 24, 2003, from http://www.ci.el-paso.tx.us/econdev/eddepeo3a.htm

Connors, N. (1992). Wellness promotes healthier employee: Companies see decreases in turnover, and sometimes lower medical costs, as a result of wellness programs. Business and Health, 10(3), 6671.

Fowler, B. A., \& Risner, P. B. (1994). A health promotion program evaluation in a minority industry. The Journal of the Association of Black Nursing Faculty, 5(3), 72-76.

Glasgow, R. E., McCaul, K. D. \& Fisher, K. J. (1993). Participation in worksite health promotion: A critique of the literature and recommendations for future practice. Health Education Quarterly, 20, 391-408.

Kizer, K. W., Pelletier, K. R., \& Fielding, J. E. (1995). Worksite health promotion programs and health care reform. The Western Journal of Medicine, 32, 461-468.

Mason, J. C. (1982). Healthy equals happy plus productive. Management Review, 81(7), 33-38.

Michie, D. A. (1992). Paso del Norte regional economy socioeconomic profile. University of Texas at El Paso, The Institute for Manufacturing and Materials Management, Burges Hall.

Prata, K. (1993). Worksite wellness. American Fitness, 11(3), 18-21.

Reardon, J. (1998). The history and impact of worksite wellness. Nursing Economics, 16(3), 117-121.

Sorensen, G., Glasgow, R. E. \& Corbett, K. (1990). Involving work sites and other organizations. In N. Bracht (Ed.), Health Promotion at the Community Level (pp. 158-184). Newbury Park: Sage Publications.

Stead, B. A. (1994). Worksite health programs: A significant cost-cutting approach. Business Horizons, 37(6), 73-79.

Stokols, D., Pelletier, K. R., \& Fielding, J. E. (1995). Integration of medical care and worksite health promotion. The Journal of the American Medical Association, 273, 1136-1143.

United States Census Bureau. (2002). United States Census 2000. Retrieved November 24, 2003, from http://factfinder.census.gov/servlet/BasicFactsServlet

Wellness Councils of America (1994). The healthy workplace: Creating wellness for a profitable workplace, 16(8), 77-89.

\section{Acknowledgements}

This project was supported by a grant from the Paso del Norte Health Foundation. Its contents are solely the responsibility of the author and do not necessarily represent the official views of the Paso del Norte Health Foundation.

\author{
Author Information \\ Sharon E. Thompson, MPH, Ph.D., CHES \\ Assistant Professor \\ Health Science Program \\ The University of Texas at El Paso \\ 1101 North Campbell Street \\ El Paso, Texas 79902 \\ Ph. (915) 747-7221 \\ Fax. (915) 747-7207 \\ E-mail: sthompson@utep.edu
}

